\title{
Prevalence and prognosis of COPD in critically ill patients between 1998 and 2008
}

\author{
Georg-Christian Funk*, Peter Bauer*, Otto Chris Burghuber*, Andreas Fazekas*, \\ Sylvia Hart|*, Helene Hochrieser ${ }^{\#}$, Rene Schmutz and Philipp Metnitz
}

ABSTRACT: The epidemiology of chronic obstructive pulmonary disease (COPD) in critically ill patients is largely unknown. The aims of the study were: 1) to determine whether COPD, either as the cause of intensive care unit (ICU) admission or as a comorbid condition, is an independent risk factor for increased morbidity and mortality; and 2) to investigate time trends in proportion and outcome of acute respiratory failure in patients with COPD admitted to ICUs.

Prospectively recorded data from 194453 adults consecutively admitted to 87 Austrian ICUs over a period of 11 years (1998-2008) were retrospectively analysed.

COPD was present in $8.6 \%$ of all patients. The risk-adjusted mortality of patients with COPD was higher than in patients without COPD. The presence of COPD was an independent risk factor for increased mortality and was associated with prolonged mechanical ventilation and prolonged weaning. During the course of the 11 years, the proportion of acute respiratory failure due to COPD increased by about two-thirds, and the use of noninvasive ventilation within the COPD cohort more than doubled. Simultaneously, the risk-adjusted mortality of patients with COPD improved.

In critically ill patients, the presence of COPD is increasing and is an independent risk factor for mortality and morbidity.

KEYWORDS: Epidemiology, mechanical ventilation, noninvasive ventilation, respiratory care unit, weaning centre

$\mathbf{T}$ he global prevalence of chronic obstructive pulmonary disease (COPD) is increasing dramatically [1]. In Austria one out of four persons over the age of 40 years has COPD [2]. However, it is not known whether the proportion of acute respiratory failure (ARF) due to COPD is also increasing. Moreover, it is not well established whether the presence of COPD is a risk factor for prolonged mechanical ventilation, prolonged weaning and an eventual poor prognosis. This information would be valuable for the planning of resources in respiratory care units and weaning facilities.

The two most commonly used scores for estimation of mortality risk in the intensive care unit (ICU), the Simplified Acute Physiology Score (SAPS) II and the Acute Physiology and Chronic Health Evaluation (APACHE) score, do not include COPD as a chronic condition [3,4]. This may be due to difficulties in defining COPD in the databases that were used for the development of these scores. In contrast, some of the cardiac surgical models do use COPD as a risk factor, because it is a clear risk factor for both morbidity and mortality in these populations $[5,6]$.
We speculated that COPD would be an increasing problem in critically ill patients and would be associated with increased morbidity and mortality. The aims of this retrospective record review were: 1 ) to determine whether COPD, either as the cause of ICU admission or as a comorbid condition, is an independent risk factor for increased morbidity and mortality; and 2) to investigate time trends in proportion and outcome of ARF in patients with COPD treated in ICUs.

\section{MATERIALS AND METHODS \\ Database}

Data were collected by the Austrian Centre for Documentation and Quality Assurance in Intensive Care Medicine (ASDI) (www.asdi.at), a nonprofit organisation which has established an intensive care database and benchmarking project [7]. The SAPS II, used to quantify the severity of illness upon ICU admission and to estimate the risk of dying, was used [3]. The score is calculated from 12 routine physiological measurements during the first $24 \mathrm{~h}$ after admission, information about previous health status and some information obtained at admission. The prospectively collected data included sociodemographic information,
AFFILIATIONS

*Ludwig Boltzmann Institute for COPD and Respiratory Epidemiology, Dept of Respiratory and Critical Care Medicine, Otto Wagner Spital, Vienna,

"Dept of Medical Statistics, Medical University of Vienna, Vienna, and

"Dept of Anaesthesiology and General Intensive Care Medicine, Medical University of Vienna, Vienna, Austria.

CORRESPONDENCE

P. Metnitz

Dept of Anaesthesiology and General Intensive Care Medicine

Medical University of Vienna

Austria

Währingergürtel 18-20

A-1090 Vienna

Austria

E-mail: philipp@metnitz.biz

Received:

Dec 272011

Accepted after revision:

July 132012

First published online:

Sept 272012 
such as age, sex and comorbid conditions; causes of ICU admission according to a pre-defined list of medical and surgical diagnoses [8]; length of ICU and hospital stay; and outcome data, including survival status at ICU and hospital discharge. Data collectors coded diagnoses on the basis of documentation in the patients' medical records. A total of 194453 patients were analysed (fig. 1). The study protocol was approved by institutional review. Since no additional interventions were performed and no individual data were analysed, the need for informed consent was waived.

\section{Inclusion and exclusion criteria}

We included all patients from ICUs that participated in the ASDI project. We excluded patients who were admitted before January 1, 1998 or after December 31, 2008, patients from ICUs with insufficient data quality, patients who lacked clear identification or who were documented twice, patients with records that lacked an entry in the field "hospital outcome", and patients without a valid SAPS II score. Due to the very low prevalence of COPD in adolescents, patients aged $<18$ years were excluded [1]. In patients who were admitted more than once, only the first admission was used.

\section{Data quality}

To assess the reliability of data collection, we sent an independent observer to each unit to obtain SAPS II data from the clinical charts of a random sample of patients. Variance-component

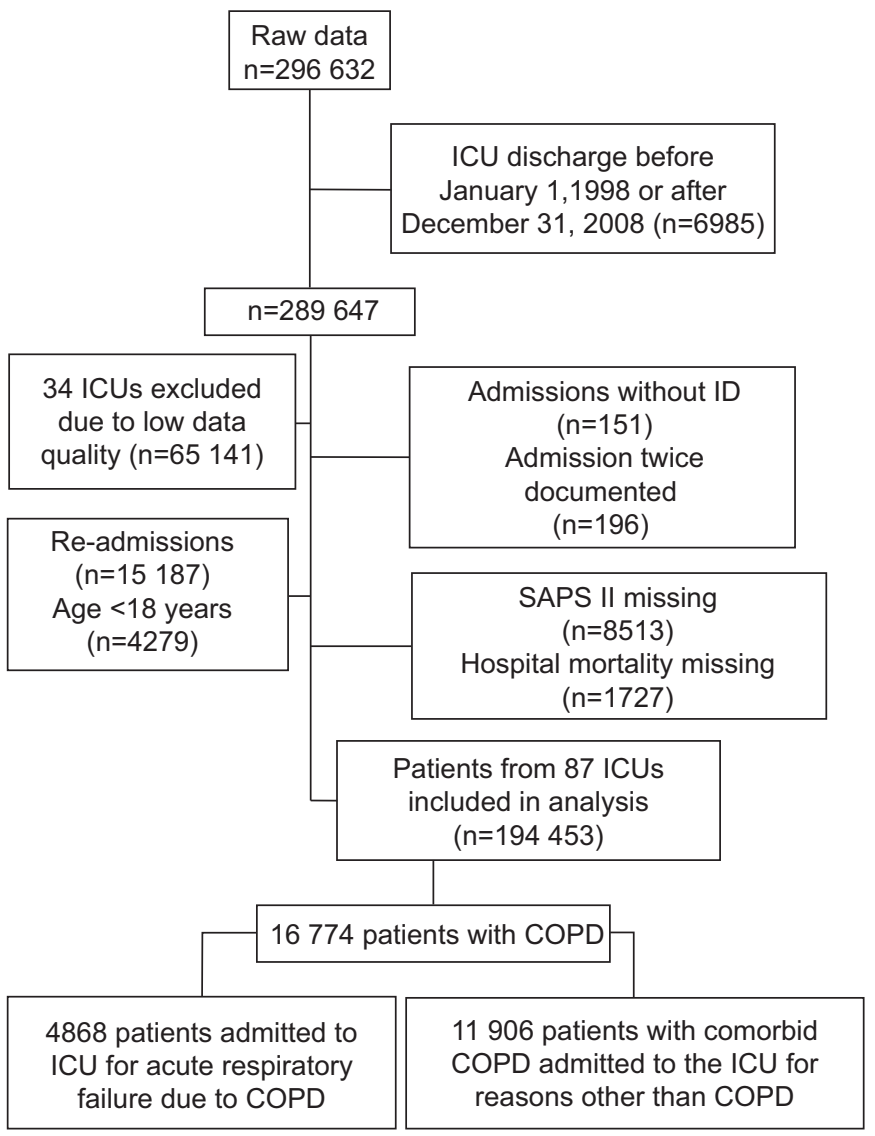

FIGURE 1. Study flow chart. ICU: intensive care unit; SAPS: Simplified Acute Physiology Score; COPD: chronic obstructive pulmonary disease. analyses with the random factors "units", "patients within units" and "observers within units" were performed (Varcomp procedure; SAS Institute, Cary, NC, USA), as previously described [7]. To assess completeness of documentation, we also calculated the number of missing variables for the SAPS II score. Additional details have been reported elsewhere [7].

Data quality was satisfactory with respect to both completeness of records and inter-rater variability. The median number of missing variables necessary for the calculation of the SAPS II was 0 (interquartile range $0-2$ ). Inter-rater quality control indicated an excellent grade of agreement: for all tested variables, practically no deviations between the observers were detected, the contribution to the variability being $<1 \%$.

\section{Definition of the COPD cohort}

The cohort of COPD patients consisted of patients who had ARF due to COPD as the main reason for ICU admission (i.e. ICU admission because of COPD) and of patients who had a history of comorbid COPD but who did not have ARF due to COPD as the main reason for ICU admission (i.e. ICU admission with comorbid COPD). The diagnosis "COPD" was based on the judgment of the intensive-care physicians, and included information provided by the patient, relatives or healthcare professionals and information in previous medical reports (e.g. pulmonary function tests) and information about previous COPD medication. In addition, physical, radiographic and/or physiological findings that were suggestive of COPD also influenced the judgment.

\section{Outcomes}

Vital status on ICU discharge and hospital discharge defined ICU and hospital mortality, respectively. The total number of days spent in the ICU defined the ICU length of stay. The number of the ventilator-free days in a period of 28 days was defined by the sum of days that patients spent without an invasive airway or a noninvasive interface up to day 28 after ICU admission. Patients who had an invasive airway for $\geqslant 21$ consecutive days where considered to have prolonged mechanical ventilation [9]. Patients were considered to have prolonged weaning when they were breathing through an invasive or noninvasive airway with ventilator assistance (pressure support ventilation, proportional assist ventilation or continuous positive airway pressure) for $\geqslant 7$ consecutive days [10]. To fulfill readiness for weaning, patients needed to be free of vasoactive drugs and renal replacement therapy for 7 days [10].

In addition, we recorded the number of patients who received noninvasive ventilation at least once at any time during their stay in the ICU, the number of patients who had any kind of invasive airway at least once at any time during their stay in the ICU and the number of patients who required tracheotomy during their stay in the ICU.

\section{Statistical analysis}

Statistical analysis was performed using SAS software version 9.2 (SAS Institute, Cary, NC, USA). Unless otherwise stated, descriptive results are expressed as median (interquartile range). The Chi-squared test and the Mann-Whitney U-test were used for comparisons of the groups. Observed-toexpected mortality ratios were calculated by dividing the 
number of observed deaths per group by the number of SAPS II-predicted deaths per group. Confidence intervals of $95 \%$ were calculated according to a formula described by HOSMER and LEMESHOW [11]. Logistic regression analysis was used to explore the influence of COPD (as cause of admission or as comorbid condition) on mortality. Vital status at hospital discharge (hospital mortality) was used as the dependent variable. To exclude case mix factors as possible confounders, the model was adjusted for the following predefined variables: male sex, SAPS II score, and all interactions between COPD, male sex and SAPS II score. The Brier score and the area under the receiver operator characteristic curve were used to evaluate the fit of the model. To assess the significance of time trends, a time variable was added. To consider dependencies between patients within ICUs, we used the ICU as a random factor in the method of general estimation equations. A p-value $<0.01$ was chosen as the significance level for relevant influence factors to be discussed to avoid overfitting. In addition, simple logistic regression with time as a covariate was used for the exploration of trends in proportion data over time. Cumulative incidence curves were calculated using competing risk methodology (cuminc function in $\mathrm{R}$ (University of Vienna, Vienna, Austria)) [12].

\section{RESULTS}

\section{Characteristics of the overall ICU cohort}

A total of 194453 consecutively admitted ICU patients from 87 ICUs were included in the cohort. The types of ICU admissions were medical and neurological disorders in 93990 (48.6\%) patients, elective surgery in $63627(32.9 \%)$ patients and emergency surgery in 35911 (18.6\%) patients. Median ICU length of stay was 3 days (range 2-6 days). Overall ICU mortality was $11.3 \%$, and overall hospital mortality was $16.6 \%$. Characteristics of patients with and without COPD are shown in table 1. Comorbidities and main causes of ICU admission are shown in table 2.

\section{Epidemiology of COPD upon ICU admission}

Altogether, $16774(8.6 \%)$ of all of the patients admitted to the ICUs had COPD. Of the 16774 COPD patients, 4868 (29\%) patients were admitted because of ARF due to COPD. This corresponded to $2.5 \%$ of all patients. The other 11906 patients (71\% of all COPD patients) had COPD as a comorbid condition but not as the main cause of ICU admission (fig. 1).

The characteristics of patients with comorbid COPD compared to patients without COPD are shown in table 1. Overall, COPD

TABLE 1

Associations of chronic obstructive pulmonary disease (COPD) as the cause of admission or as a comorbid condition with patient comorbidities and diagnoses at intensive care unit (ICU) admission and with outcome

\section{Patients admitted to the ICU without COPD}

Patients admitted to the ICU

because of acute respiratory failure due to COPD

\begin{tabular}{|c|c|c|c|c|}
\hline Subjects & 177679 & 4868 & 11906 & \\
\hline \multicolumn{5}{|l|}{ Demographic characteristics } \\
\hline Age years & $62.5 \pm 17.3$ & $67.2 \pm 12.1$ & $68.7 \pm 13.2$ & $<0.0001^{\#, \bullet,+}$ \\
\hline Male patients & $101674(57.4)$ & $3087(63.5)$ & $7191(60.5)$ & $<0.0001^{\# \cdot \varphi_{1}+}$ \\
\hline \multicolumn{5}{|l|}{ Type of admission } \\
\hline Medical & 83991 (47.5) & $4868(100.0)$ & $5131(43.2)$ & $<0.0001^{\# \cdot \varphi_{+}+}$ \\
\hline Scheduled surgical & $59604(33.7)$ & $0(0.0)$ & $4023(33.8)$ & $<0.0001^{\#,+}$ \\
\hline Unscheduled surgical & $33177(18.8)$ & $0(0.0)$ & $2734(23.0)$ & $<0.0001^{\# \cdot \varphi_{1}+}$ \\
\hline \multicolumn{5}{|l|}{ Risk upon ICU admission } \\
\hline SAPS II original model & $27.0(18.0-40.0)$ & $41.0(31.0-55.0)$ & $34.0(24.0-48.0)$ & $<0.0001^{\#, \bullet,+}$ \\
\hline SAPS II predicted mortality & $34202(19.3)$ & $1746(35.9)$ & 3201 (26.9) & $<0.0001^{\#, \bullet,+}$ \\
\hline \multicolumn{5}{|l|}{ Outcome } \\
\hline ICU length of stay days & $3.00(2.00-6.00)$ & $6.00(3.00-14.00)$ & $4.00(2.00-9.00)$ & $<0.0001^{\#, \varphi_{1}+}$ \\
\hline Observed ICU mortality & $18907(10.6)$ & $1017(20.9)$ & $2127(17.9)$ & $<0.0001^{\# \cdot \varphi_{+}+}$ \\
\hline Post-ICU mortality & $8599(4.8)$ & $532(10.9)$ & $1007(8.5)$ & $<0.0001^{\#, \varphi_{+}+}$ \\
\hline Observed hospital mortality & $27520(15.5)$ & $1549(31.8)$ & $3134(26.3)$ & $<0.0001^{\#, \cdot,+}$ \\
\hline $\begin{array}{l}\text { Ratio of observed to expected } \\
\text { mortality }(95 \% \mathrm{Cl})\end{array}$ & $0.805(0.797-0.812)$ & $0.887(0.857-0.918)$ & $0.979(0.956-1.003)$ & \\
\hline $\begin{array}{l}\text { Patients with prolonged } \\
\text { mechanical ventilation }\end{array}$ & 22961 (12.9) & $1675(34.4)$ & $2406(20.2)$ & $<0.0001^{\#, \varphi_{1}++}$ \\
\hline Patients requiring tracheotomy & $10516(5.9)$ & $966(19.8)$ & $1424(12.0)$ & $<0.0001^{\#, \cdot,+}$ \\
\hline Patients with prolonged weaning & $4140(2.3)$ & $452(9.3)$ & $462(3.9)$ & $<0.0001^{\# \cdot \varphi_{,+}+}$ \\
\hline
\end{tabular}

Patients admitted to the ICU with comorbid COPD

\section{p-value}

$$
\begin{aligned}
& <0.0001^{\#, \cdot,+} \\
& <0.0001^{\#, \bullet,+} \\
& <0.0001^{\#,+} \\
& 0.0001^{\# \cdot 9} \\
& <0.0001^{\#, \cdot,+} \\
& 0.0001^{\#, \cdot,+} \\
& <0.0001^{\# \cdot \text { : }+} \\
& <0.0001^{\#, 9,+} \\
& <0.0001^{\#,+} \\
& <0.0001^{\#, \varphi_{\text {, }}+} \\
& <0.0001^{\#, 9,+} \\
& 0.0001^{\#, \varphi_{,+}}
\end{aligned}
$$

Data are presented as n, mean + SD, n (\%) or median (interquartile range), unless otherwise stated. SAPS: Simplified Acute Physiology Score. p-values for comorbidites and diagnoses at admission obtained by Chi-squared test and for outcome obtained by Mann-Whitney U-test. \# : significant difference between patients without COPD patients with comorbid COPD in post hoc analysis. ${ }^{+}$. significant difference between patients admitted to the ICU because of acute respiratory failure due to COPD versus patients with comorbid COPD in post hoc analysis. 
TABLE 2 Comorbidities and main causes of intensive care unit (ICU) admission for patients with chronic obstructive pulmonary disease (COPD) as cause of admission or as a comorbid condition and for patients without COPD

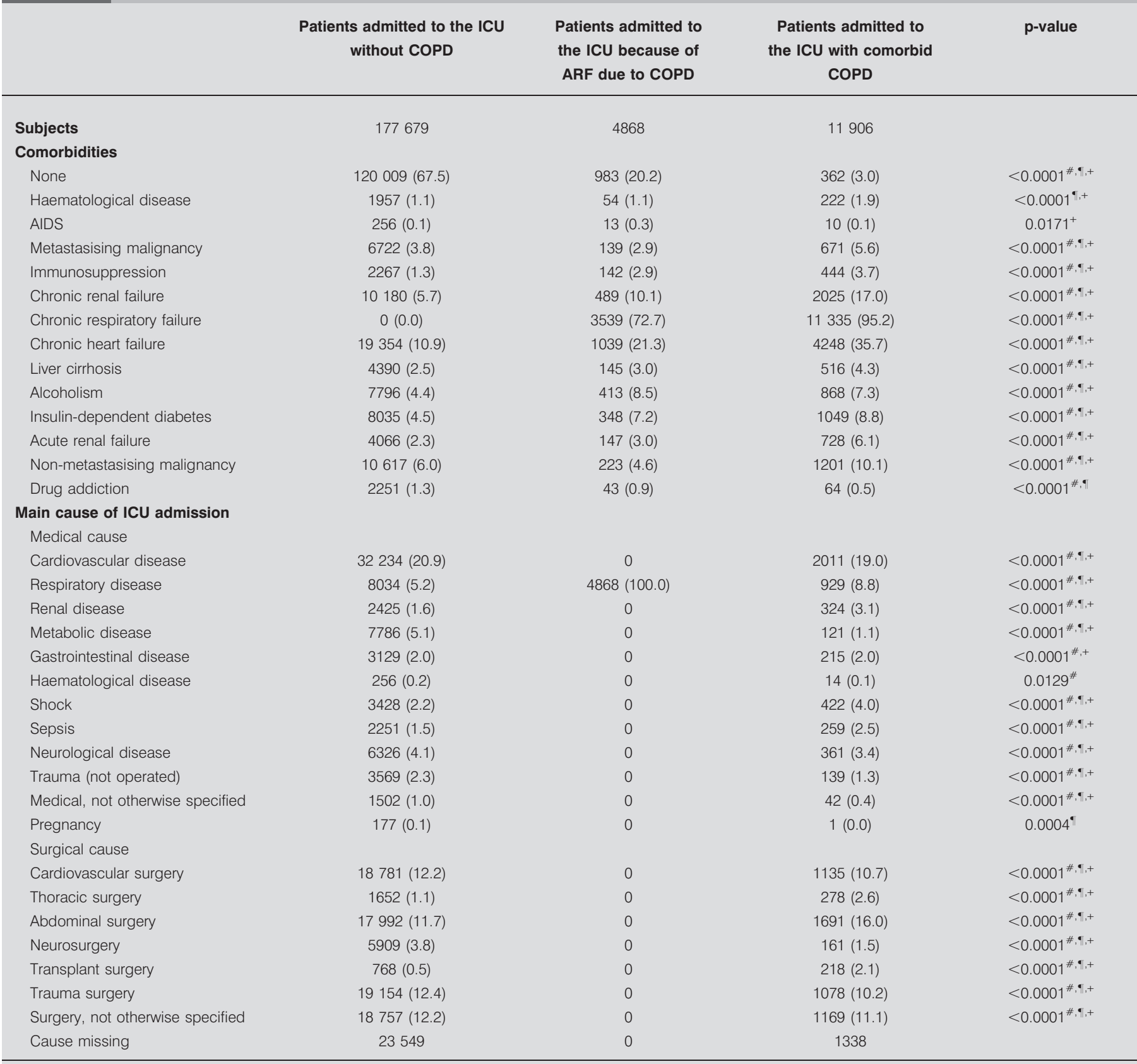

Data are presented as $n$ or $n(\%)$, unless otherwise stated. p-values obtained by Chi-squared test. ARF: acute respiratory failure. ${ }^{\#}$ : significant difference between patients without COPD versus patients admitted to the ICU because of acute respiratory failure due to COPD in post hoc analysis; ${ }^{\prime}$ : significant difference between patients without COPD versus patients with comorbid COPD in post hoc analysis; ${ }^{+}$: significant difference between patients admitted to the ICU because of acute respiratory failure due to COPD versus patients with comorbid COPD in post hoc analysis.

patients were older, more likely to be male and had more comorbidities, such as congestive heart failure, diabetes and chronic renal failure (table 2).

\section{Outcome in patients with and without COPD}

Compared to patients without COPD, the adjusted mortality, as assessed by the ratio of observed to expected mortality, was higher in patients admitted because of ARF due to COPD and even higher in patients with comorbid COPD (table 1). The probability of dying was highest in patients who were admitted because of ARF due to COPD. Patients with comorbid COPD were more likely to die compared to patients without COPD, e.g. on day 20 after ICU admission the probability of being dead was $22 \%, 19 \%$ and $12 \%$ in patients 


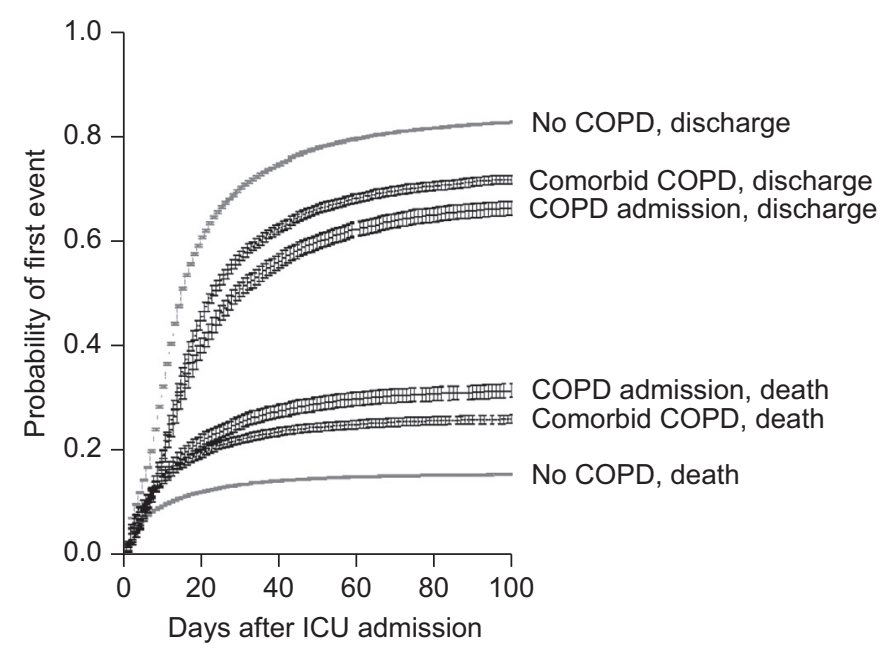

FIGURE 2. Cumulative probability of discharge or death in patients with no chronic obstructive pulmonary disease (COPD) versus patients with comorbid COPD versus patients who were admitted because of acute respiratory failure due to COPD together with point-wise $95 \%$ confidence intervals. ICU: intensive care unit.

who were admitted because of ARF due to COPD, in patients with comorbid COPD and in patients without COPD, respectively $(\mathrm{p}<0.0001)$ (fig. 2). In line with this observation, the probability of being discharged alive was lowest in patients who were admitted because of ARF due to COPD. Patients with comorbid COPD were less likely to be discharged alive compared to patients without COPD, e.g. on day 20 after ICU admission the probability of being discharged alive was $40 \%$, $46 \%$ and $61 \%$ in patients who were admitted because of ARF due to COPD, in patients with comorbid COPD and in patients without COPD, respectively $(\mathrm{p}<0.0001)$ (fig. 2$)$. The cumulative probability of discharge or death in patients with COPD versus patients without COPD is shown in figure 2.

Compared to patients without COPD, prolonged weaning, prolonged mechanical ventilation and tracheotomy were more common in patients with comorbid COPD and even more common in patients admitted because of ARF due to COPD (table 1).
The proportion of ventilator free-days within 28 days of ICU admission in patients without COPD, in patients with comorbid COPD and in patients admitted because of ARF due to COPD was $58 \%, 47 \%$ and $31 \%$, respectively $(p<0.0001)$.

COPD as an independent risk factor for increased mortality After adjustment for the predefined variables (male sex, SAPS II and year), the presence of COPD was independently associated with increased mortality (table 3). Independent of the other risk factors, there was a negative significant trend for the year, which indicated less mortality later in the study (OR 0.973 ). This corresponds to a reduction in mortality of $2.7 \%$ per year during the observation period. The influence of the other risk factors was small in magnitude compared to the influence of COPD, SAPS II and time.

Sex has neither a relevant influence on mortality nor does it show any relevant interaction. However, for patients who were admitted to the ICU because of ARF due to COPD, there was a relevant interaction with the SAPS II score, indicating that if both influence factors are present the risk is lower than simply adding their individual contributions. A Brier score of 0.1063 and an area under the receiver operator characteristic curve of 0.8597 were calculated for the model fit.

\section{Epidemiology and outcome of COPD patients over time}

The overall prevalence of COPD in critically ill patients decreased slightly over the course of the years studied (fig. 3). However, the overall proportion of ARF due to COPD as the main cause of ICU admission increased over time by approximately two-thirds (from $1.8 \%$ to $3.0 \%$ ) (fig. 3).

The risk-adjusted mortality of both ICU patients with comorbid COPD and of patients admitted to the ICU because of ARF due to COPD decreased substantially over time (fig. 4). This reduction was due to both a decrease in mortality and a simultaneous increase of risk as assessed by the SAPS II score (data not shown).

No time trend was found for the proportion of prolonged mechanical ventilation and tracheotomy in patients admitted because of ARF due to COPD (fig. 5). However, the proportion of prolonged weaning increased over time in patients admitted

TABLE 3 Determinants of hospital mortality among intensive care unit (ICU)-dependent critically ill patients

\begin{tabular}{lcc} 
Variable & OR (95\% CI) & p-value \\
\hline Intercept & \\
COPD as cause of ICU admission & $3.16(2.58-3.87)$ \\
COPD as comorbidity & $1.39(1.22-1.60)$ \\
Male sex & $1.05(0.99-1.12)$ \\
SAPS II & $1.07(1.07-1.07)$ \\
Interaction between COPD as cause of ICU admission and sex & $1.09(0.99-1.21)$ \\
Interaction between COPD as comorbidity and sex & $1.06(0.98-1.14)$ \\
Interaction between COPD as cause of ICU admission and SAPS II & $0.98(0.97-0.98)$ \\
Interaction between COPD as comorbidity and SAPS II & $1.00(0.99-1.00)$ \\
Interaction between sex and SAPS II & $1.00(1.00-1.00)$ \\
Year & $0.97(0.96-0.98)$ \\
\hline
\end{tabular}

COPD: chronic obstructive pulmonary disease; SAPS: Simplified Acute Physiology Score. 


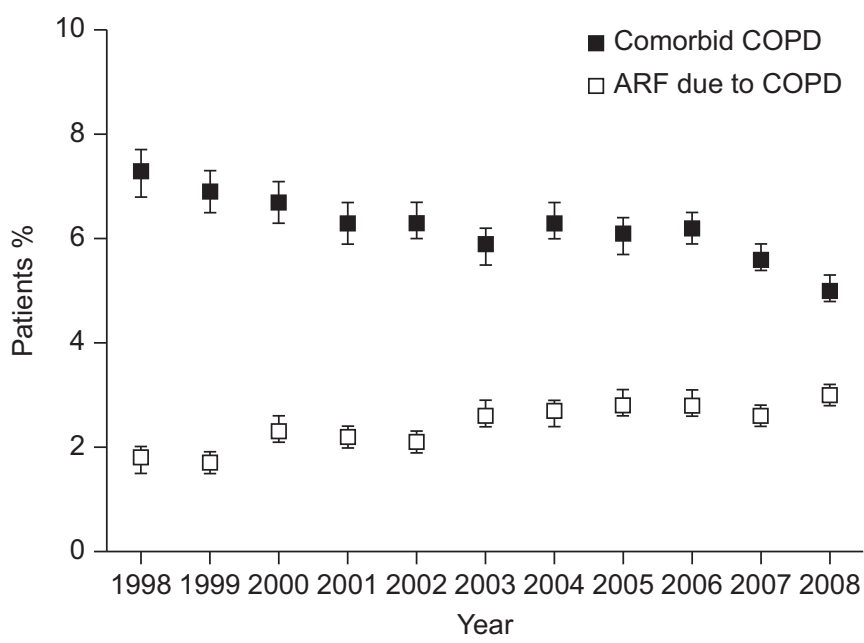

FIGURE 3. Time trend of the prevalence of comorbid chronic obstructive pulmonary disease (COPD) at the time of intensive care unit admission (OR 0.97; $p<0.0001$ ) and acute respiratory failure (ARF) due to COPD (OR 1.050; $p<0.0001$ ) of the whole cohort $(n=194453)$. The $p$-values result from the testing of a linear time trend in a logistic regression model. Error bars represent 95\% confidence intervals.

because of ARF due to COPD (fig. 5). The proportion of COPD admissions who received noninvasive ventilation at least once during their stay in the ICU more than doubled from $14 \%$ in 1998 to $50 \%$ in 2008 ( $p<0.0001$ ) (fig. 6 ). This was paralleled by a small but significant decrease in the use of invasive ventilation in patients admitted because of ARF due to COPD $(62 \%$ in 1998 versus $58 \%$ in 2008; $\mathrm{p}=0.0032)$.

\section{DISCUSSION}

The presence of COPD was found to be a risk factor for increased mortality and morbidity in critically ill patients. During the 11 years 1998-2008, the risk-adjusted mortality of these patients improved.

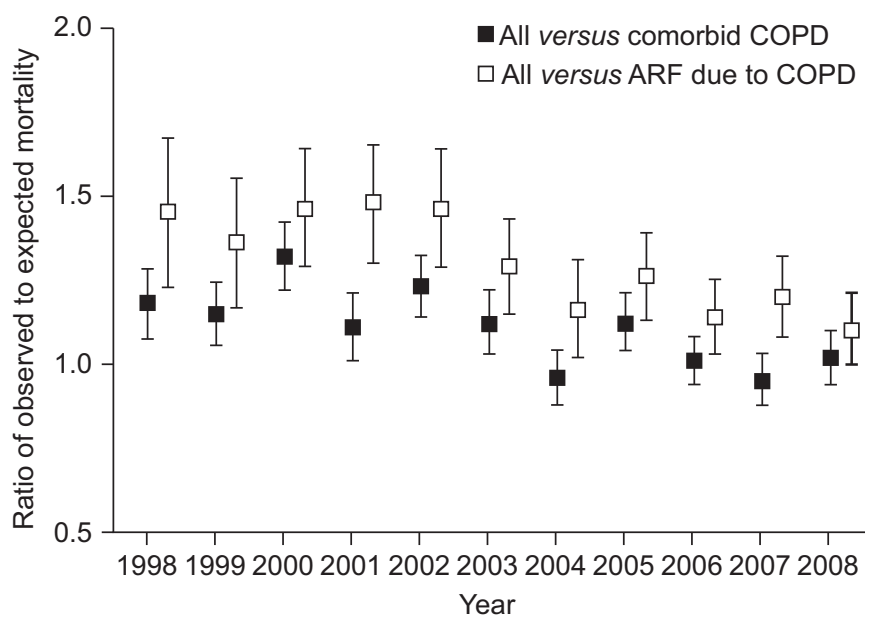

FIGURE 4. Risk-adjusted mortality over time as assessed by the ratio of observed to expected mortality of all intensive care unit (ICU) patients with comorbid chronic obstructive pulmonary disease (COPD) and patients with acute respiratory failure (ARF) due to COPD as the main cause of ICU admission. Error bars represent $95 \%$ confidence intervals.

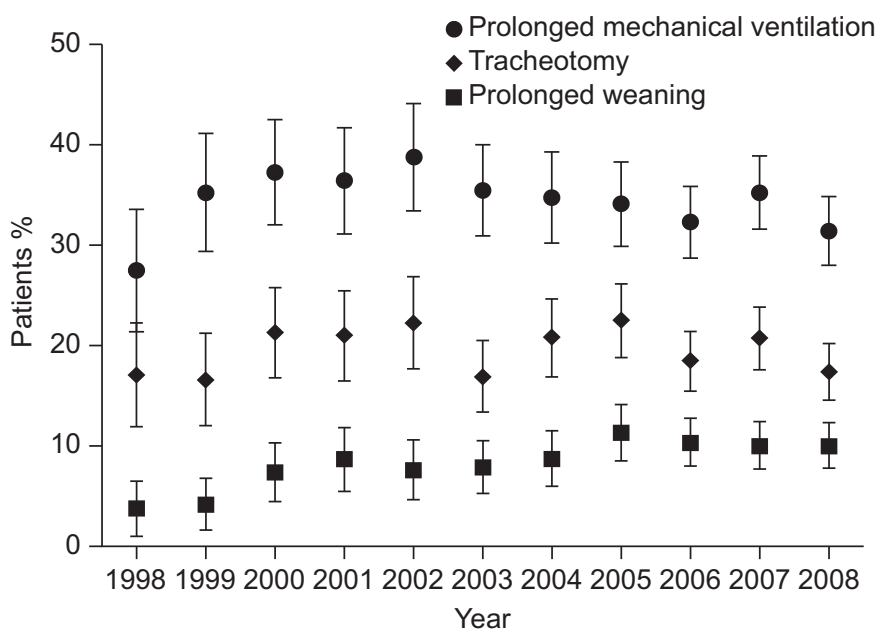

FIGURE 5. Proportion of patients with prolonged mechanical ventilation (OR 0.99 ; $p=0.28)$, tracheotomy (OR 1.00; $p=0.90)$ and prolonged weaning (OR 1.07; $p<0.0001)$ in patients admitted because of acute respiratory failure due to chronic obstructive pulmonary disease $(n=4868)$. The $p$-values result from the testing of a linear time trend in a logistic regression model. Error bars represent 95\% confidence intervals.

This is the largest study of the epidemiology and outcome of patients with COPD treated in ICUs. Our study is also the first to assess time trends in morbidity and mortality in critically ill patients with COPD. Our finding of an improvement in prognosis over time is in concordance with the recently observed mortality reduction observed in stable patients with COPD [13]. These improvements may be due to an improved initiation of relevant inhalation medication outside the hospital and a focus on rehabilitation among severe COPD patients during the last decade.

Several longitudinal studies have demonstrated that COPD patients who require mechanical ventilation have a very poor prognosis [14-16]. However, no large study has compared whether the presence of COPD is an independent risk factor

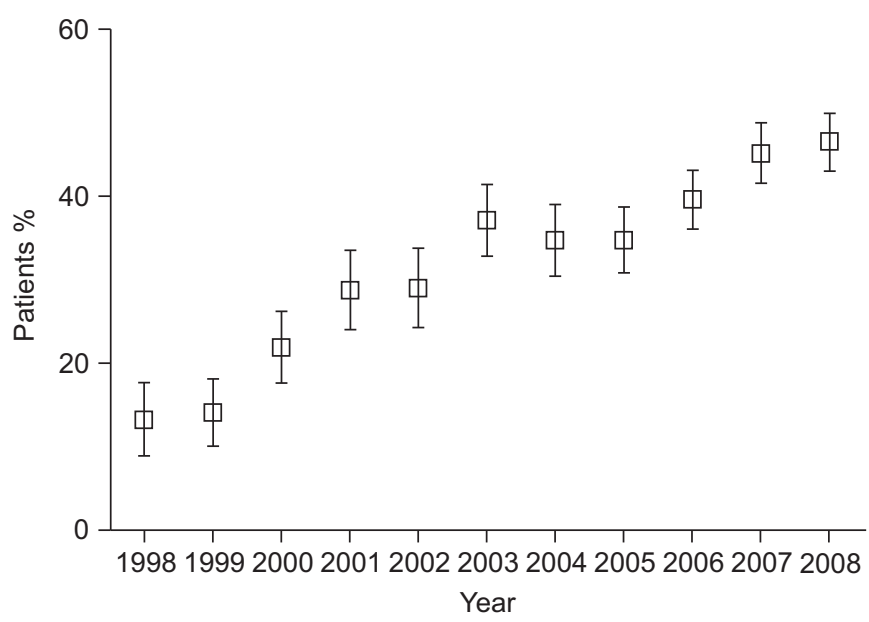

FIGURE 6. Proportion of patients admitted because of acute respiratory failure due to chronic obstructive pulmonary disease $(n=4868)$ who received noninvasive ventilation at least once during their stay in the ICU (OR 1.165; $p<0.0001$ in a linear time trend in a logistic regression model). Error bars represent 95\% confidence intervals. 
for increased mortality. In one study of weaning classification, chronic respiratory failure was associated with prolonged weaning [17]. In another recent study, COPD was associated with prolonged weaning in a univariable analysis but not after multivariable adjustment for confounders [18]. In the largest weaning register of 2714 patients, COPD was an uncommon reason to institute mechanical ventilation (120 patients, $4 \%$ ) [19]. Not surprisingly, no association between COPD and prolonged weaning was detected.

Why was the risk-adjusted mortality (assessed by the ratio of observed to expected mortality) higher in patients with comorbid COPD than in patients admitted because of ARF due to COPD? This may be explained by the negative interaction between SAPS II and "admission because of COPD", indicating that influence factors do not act in an additive way. We suppose that the strong influence of the Glasgow Coma Scale on the SAPS II score overestimates mortality risk in the presence of reversible hypercapnic encephalopathy. This explanation remains speculative due to the absence of arterial carbon dioxide tension values upon ICU admission and the absence of serial recordings of Glasgow Coma Scale.

There are some limitations to the data. The diagnosis of COPD used in this study was based on previous history and on clinical findings. A systematic diagnostic work-up, including spirometry in survivors, was not performed. Therefore, it can be assumed that some of the patients classified with COPD actually did not have COPD but another kind of lung disease, such as asthma. However, the true proportion of COPD in critically ill patients requiring ICU therapy may have been underestimated because not all patients who truly had COPD were classified with this condition. In contrast, if a substantial number of patients with conditions other than COPD (e.g. heart failure) were misclassified as having COPD, the real influence of COPD on mortality might be overestimated. Although not systematically validated, clinical judgment for diagnosing COPD in critically ill patients has been used in comparable studies $[6,15]$. Moreover, two major scores for estimating risk prior to cardiac surgery include COPD defined by clinical definitions such as "long-term use of bronchodilators or steroids for lung disease" or "clinical history plus use of bronchodilating agents" $[5,6]$. The observation that COPD was associated with increased morbidity mortality in these risk models increases the validity of the definition of COPD used in this study.

The absence of lung function data and detailed information about the functional capacities of the patients did not allow for the discrimination of factors that were responsible for the poor outcome (e.g. reduced exercise capacity or dyspnoea prior to critical illness).

Because the readiness for weaning according to pre-specified criteria [10] was not documented prospectively, we relied on the available indicators for this condition. We reasoned that the combination "absence of vasoactive drugs", "absence of renal replacement therapy" and "presence of an assisted ventilatory mode" was a good surrogate indicator that the patient was actually ready to wean. However, not all of the criteria that are required for fulfilling readiness to wean could be assessed. For example, adequate coughing and adequate mentation were not part of our epidemiological database. Therefore, some of the patients classified as being in the process of weaning in this study may have actually been awaiting readiness to wean in reality. Therefore, the real duration of weaning may have been shorter than reported in the paper.

Since re-admissions were not analysed, we assumed that patients discharged from the ICU within 28 days of the first ICU admission did not require further mechanical ventilation. Therefore, the real proportion of ventilator-free days in patients re-admitted to an ICU within 28 days was probably lower than that reported. This bias affects all three groups of patients identically. Hence, the absolute percentages of ventilator-free days should be interpreted with caution; however, betweengroup differences are probably valid.

There may be a variety of reasons for the association of COPD with increased mortality. Impaired respiratory mechanics and reduced capacity of the ventilatory muscle pump are associated with weaning failure and an increased duration of mechanical ventilation in COPD [20, 21]. In turn, invasive mechanical ventilation is a risk factor for ventilator-associated pneumonia (VAP). It has been shown on an epidemiological basis that COPD is a risk factor for VAP occurrence and that COPD is related to risk factors for adverse VAP outcome, such as multidrugresistant bacteria [22]. In addition, patients with COPD had more comorbidities such as heart failure, diabetes and renal failure, which probably contribute to the increased mortality.

The presence of chronic respiratory disease is associated with an increased duration of mechanical ventilation and prolonged weaning [17]. Respiratory muscle weakness, weakness of the limbs muscles and chronic extrapulmonary organ failures are the suspected mechanisms for this finding [10].

The observed improvement of risk-adjusted mortality over time was due to both an increase in the severity of illness and a decrease in mortality. The increase of the severity of illness suggested that the proportion of ICU admission in very sick COPD patients increased over time. This may be due to the known benefits of noninvasive ventilation, which increasingly justified ICU treatment in patients who would not have been admitted to the ICU before [23]. The decrease in mortality over time could be attributable to avoided intubations because of the successful noninvasive ventilation and to a less aggressive sedation policy [24].

The increasing proportion of patients with prolonged weaning over time is probably explained by a parallel increase in the underlying severity of illness as assessed by an increase of SAPS II score over time. Additionally, fewer hospitals and ICU beds, as well as a more restricted access to hospitals due to an increased threshold for admission, might contribute to the observation.

This study conveys an important message for clinicians: whether COPD is present in critically ill patients is important, because it is associated with unfavourable outcomes. Although this study did not prove that specific interventions improve the outcome of patients with COPD, it does remind us that evidence-based therapies with proven benefit must not be withheld. More specifically, in a patient with COPD and ARF, every effort should be made to successfully apply noninvasive ventilation.

The increasing proportion of patients admitted to the ICU because of respiratory failure due to COPD and the prolonged 
duration of mechanical ventilation and weaning in these patients underlines the need for specialised weaning facilities. Although only $5-10 \%$ of ICU patients require prolonged mechanical ventilation, they account for $>50 \%$ of the total costs $[17-19,25]$. However, more than half of the mechanically ventilated patients who were transferred to a weaning facility could finally be weaned off the ventilator [26].

\section{Conclusions}

In conclusion, the presence of COPD was identified as a risk factor for increased mortality in critically ill patients. In addition, prolonged mechanical ventilation, prolonged weaning and tracheotomy was more common in patients with COPD. The risk-adjusted mortality observed over the course of 11 years decreased. Due to the retrospective and observational nature of the study the causes for these observations cannot be ascertained.

\section{STATEMENT OF INTEREST}

Conflict of interest information can be found alongside the online version of this article at www.erj.ersjournals.com

\section{ACKNOWLEDGEMENTS}

Information herein was presented in part at the Conference of the Austrian Society of Intensive Care Medicine in Vienna, Austria, June 15-18, 2011.

\section{REFERENCES}

1 Global Strategy for the Diagnosis, Management and Prevention of COPD. www.goldcopd.org/uploads/users/files/GOLD-Report 2013Feb13.pdf Date last accessed: February 17, 2013. Date last updated: 2011.

2 Schirnhofer L, Lamprecht B, Vollmer WM, et al. COPD prevalence in Salzburg, Austria: results from the Burden of Obstructive Lung Disease (BOLD) Study. Chest 2007; 131: 29-36.

3 Le Gall Jr, Lemeshow S, Saulnier F. A new Simplified Acute Physiology Score (SAPS II) based on a European/North American multicenter study. JAMA 1993; 270: 2957-2963.

4 Knaus WA, Wagner DP, Draper EA, et al. The APACHE III prognostic system. Risk prediction of hospital mortality for critically ill hospitalized adults. Chest 1991; 100: 1619-1636.

5 Nashef SA, Roques F, Michel P, et al. European system for cardiac operative risk evaluation (EuroSCORE). Eur J Cardiothorac Surg 1999; 16: 9-13.

6 Higgins TL, Estafanous FG, Loop FD, et al. Stratification of morbidity and mortality outcome by preoperative risk factors in coronary artery bypass patients. A clinical severity score. JAMA 1992; 267: 2344-2348.

7 Metnitz PG, Vesely H, Valentin A, et al. Evaluation of an interdisciplinary data set for national intensive care unit assessment. Crit Care Med 1999; 27: 1486-1491.

8 Metnitz PG, Steltzer H, Popow C, et al. [Definition and evaluation of a documentation standard for intensive care medicine: the ASDI (Working Group for Standardization of a documentation system for Intensive care medicine) pilot project]. Wien Klin Wochenschr 1997; 109: 132-138.
9 Bigatello LM, Stelfox HT, Berra L, et al. Outcome of patients undergoing prolonged mechanical ventilation after critical illness. Crit Care Med 2007; 35: 2491-2497.

10 Boles JM, Bion J, Connors A, et al. Weaning from mechanical ventilation. Eur Respir J 2007; 29: 1033-1056.

11 Hosmer DW, Lemeshow S. Confidence interval estimates of an index of quality performance based on logistic regression models. Stat Med 1995; 14: 2161-2172.

12 Gray RJ. A class of K-sample tests for comparing the cumulative incidence of a competing risk. Ann Stat 1988; 16: 1141-1154.

13 Ford ES, Mannino DM, Zhao G, et al. Changes in mortality among US adults with COPD in two national cohorts recruited from 19711975 and 1988-1994. Chest 2012; 141: 101-110.

14 Connors AF, Dawson NV, Thomas C, et al. Outcomes following acute exacerbation of severe chronic obstructive lung disease. Am J Respir Crit Care Med 1996; 154: 959-967.

15 Breen D, Churches $\mathrm{T}$, Hawker F, et al. Acute respiratory failure secondary to chronic obstructive pulmonary disease treated in the intensive care unit: a long term follow up study. Thorax 2002; 57: 29-33.

16 Afessa B, Morales IJ, Scanlon PD, et al. Prognostic factors, clinical course, and hospital outcome of patients with chronic obstructive pulmonary disease admitted to an intensive care unit for acute respiratory failure. Crit Care Med 2002; 30: 1610-1615.

17 Funk GC, Anders S, Breyer MK, et al. Incidence and outcome of weaning from mechanical ventilation according to new categories. Eur Respir J 2010; 35: 88-94.

18 Sellares J, Ferrer M, Cano E, et al. Predictors of prolonged weaning and survival during ventilator weaning in a respiratory ICU. Intensive Care Med 2011; 37: 775-784.

19 Penuelas O, Frutos-Vivar F, Fernandez C, et al. Characteristics and outcomes of ventilated patients according to time to liberation from mechanical ventilation. Am J Respir Crit Care Med 2011; 184: 430-437.

20 Jubran A, Tobin MJ. Pathophysiologic basis of acute respiratory distress in patients who fail a trial of weaning from mechanical ventilation. Am J Respir Crit Care Med 1997; 155: 906-915.

21 Carlucci A, Ceriana P, Prinianakis G, et al. Determinants of weaning success in patients with prolonged mechanical ventilation. Crit Care 2009; 13: R97.

22 Nseir S, Di PC, Soubrier S, et al. Impact of ventilator-associated pneumonia on outcome in patients with COPD. Chest 2005; 128: 1650-1656.

23 Ram FS, Picot J, Lightowler J, et al. Non-invasive positive pressure ventilation for treatment of respiratory failure due to exacerbations of chronic obstructive pulmonary disease. Cochrane Database Syst Rev 2004; 3: CD004104.

24 Girard TD, Kress JP, Fuchs BD, et al. Efficacy and safety of a paired sedation and ventilator weaning protocol for mechanically ventilated patients in intensive care (Awakening and Breathing Controlled trial): a randomised controlled trial. Lancet 2008; 371: 126-134.

25 Cohen IL, Booth FV. Cost containment and mechanical ventilation in the United States. New Horiz 1994; 2: 283-290.

26 Scheinhorn DJ, Hassenpflug MS, Votto JJ, et al. Post-ICU mechanical ventilation at 23 long-term care hospitals. Chest 2007; 131: 85-93. 